\title{
Mecánica molecular estructural para el cálculo del módulo de Young y los modos de vibración de nanotubos de carbono
}

ELECTRONIC ENGINEERING

\section{Structural molecular mechanics for calculus of Young's modulus and vibration modes of carbon nanotubes}

\author{
Ingrid M. Padilla-Espinosa*§, John M. Espinosa-Duran*, Jaime Velasco-Medina* \\ *Grupo de Bionanoelectrónica. Universidad del Valle, Cali, Colombia
}

§ingridp@univalle.edu.co,michaele@univalle.edu.co, jvelasco@univalle.edu.co

(Recibido: Marzo 09 de 2011 -Aceptado: Noviembre 19 de 2011 -Versión Final:)

\begin{abstract}
Resumen
Los nanotubos de carbono son una de las nanoestructuras más usadas para el desarrollo de nuevos nanodispositivos debido a sus excepcionales propiedades mecánicas, ópticas, químicas y eléctricas. En este trabajo, el método multiescala llamado mecánica molecular estructural se usó para estimar el módulo de Young y las frecuencias naturales de nanotubos de carbono de diferentes quiralidades y tamaños. Este método establece un enlace entre la mecánica molecular con la mecánica clásica por medio de balances de energía y permite reducir el tiempo de procesamiento en comparación con otros métodos. Los resultados de simulación obtenidos son acordes a los reportados en la literatura, en este caso, el módulo de Young varía entre 1.02 y $1.05 \mathrm{TPa}$, y las frecuencias naturales son del orden de $\mathrm{GHz}$ y son altamente dependientes de la quiralidad y de la relación longitud/radio. Teniendo en cuenta lo anterior, el método multiescala proporciona resultados de forma rápida y confiable, y es muy apropiado para el diseño de nanodispositivos, nanosensores y nanomáquinas.
\end{abstract}

Palabras Claves: nanotubos de carbono, quiralidad, módulo de Young, frecuencia natural, método multiescala, mecánica molecular estructural.

\begin{abstract}
Carbon nanotubes are one of the most used nanostructures for the development of new nanodevices due to their exceptional mechanical, chemical and electrical properties. In this work, the multiscale method called structural molecular mechanics was used to estimate Young's modulus and the natural frequencies of carbon nanotubes of different chirality and sizes. This method establishes a linkage between the molecular mechanics with classical mechanics by means energy balance and allows reducing the processing time in comparison with other methods. The obtained simulation results are in agreement with those reported in the literature, in this case, Young modulus's value varies between 1.02 a $1.05 \mathrm{TPa}$, and the natural frequencies are in the order of $\mathrm{GHz}$ and are highly dependent of chirality and the length/radius relation. Taking in account the above, the multiscale method provides fast and reliable results and is very suitable for the design of nanodevices, nanosensors and nanomachines.
\end{abstract}

Keywords: carbon nanotubes, chirality, Young's modulus, natural frequency, multiscale method, structural molecular mechanics. 


\section{Introducción}

Uno de los nanomateriales más importantes que se encuentran bajo investigación son los nanotubos de carbono CNTs (Carbon Nanotubes). Éstos se hacen cada vez más populares en la industria porque evidencian propiedades químicas, mecánicas, eléctricas, térmicas y ópticas excepcionales, Dresselhaus et al. (1995), Kalamkarov et al. (2006).

Estructuralmente, los CNTs son como una lámina de grafeno enrollada sobre sí misma, dando lugar a un cilindro (Nanotubo de carbono monocapa SWCNT, "Single Walled Carbon Nanotubes") o como varios cilindros concéntricos (Nanotubos de carbono de capa múltiple MWCNT, "Multi
Walled Carbon Nanotubes") como se muestra en la Figura 1. Cada nanotubo está formado por anillos hexagonales de carbono que se repiten, permitiendo que cada átomo tenga un enlace con otros tres átomos vecinos. Estos enlaces son muy fuertes debido a que son covalentes, por hibridaciones $\mathrm{sp}^{2}$.

Los nanotubos se caracterizan por su diámetro, longitud y quiralidad siendo esta última determinante en las propiedades. La quiralidad hace referencia a la forma en la que se da el "enrollamiento" del nanotubo y se determina geométricamente por un vector quiral OA con los parámetros $(\mathrm{n}, \mathrm{m})$ según $\mathrm{a}_{l}$ y $\mathrm{a}_{2}$, o un ángulo quiral $\theta$, mostrados en la Figura 2. De acuerdo con la quiralidad los nanotubos se clasifican en
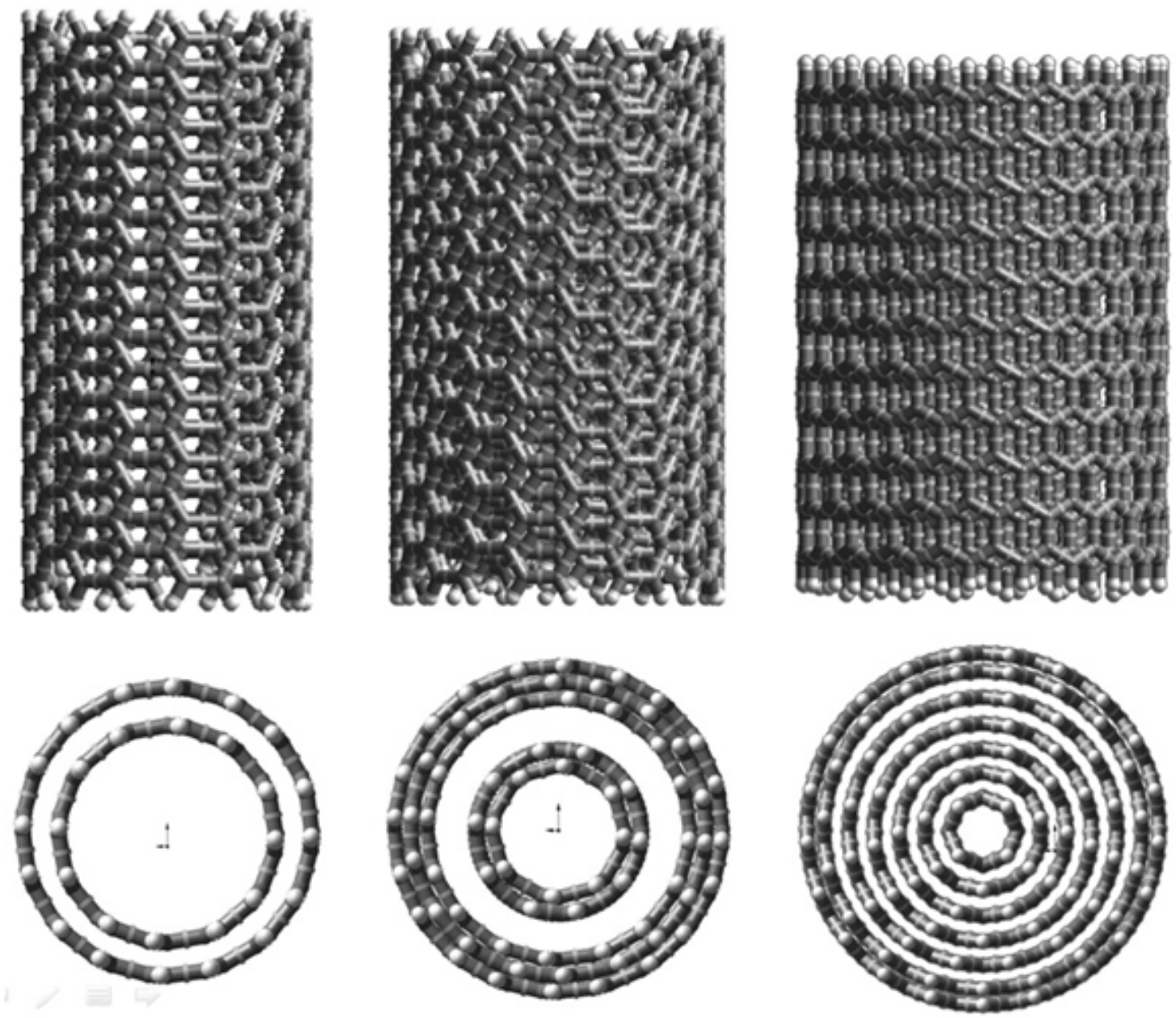

Figura 1. Nanotubos multicapa (MWCNT) con 2, 5 y 7 capas. 
"armchair", "zigzag" o quiral como se muestra en la Figura 3; cuando $n=m$ ó $\theta=30^{\circ}$ es un nanotubo armchair, cuando $\mathrm{m}=0$ ó $\theta=0^{\circ}$ es un nanotubo zigzag, cualquier otra forma es un nanotubo quiral.

Sin embargo, la realización de pruebas experimentales y los análisis teóricos cuánticos y moleculares de los nanotubos de carbono, requieren un alto costo económico y

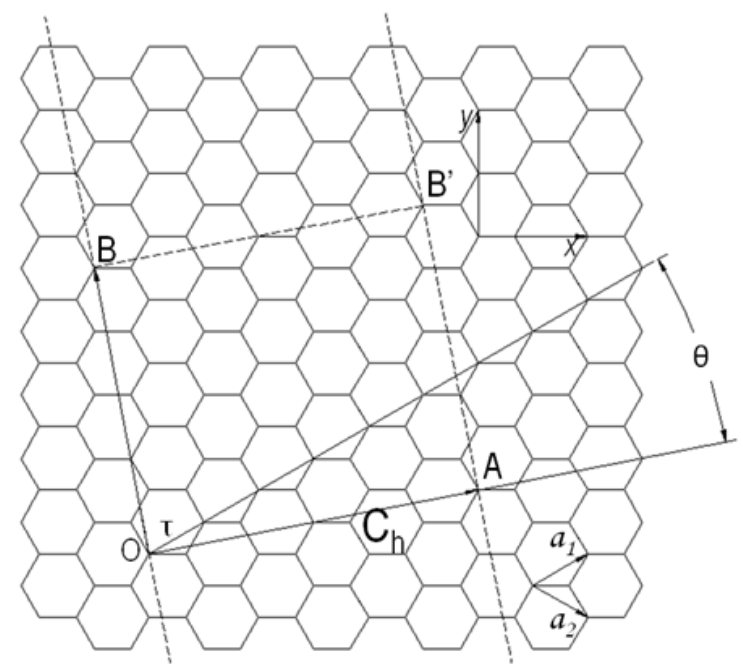

Figura 2. Vector y ángulo de quiralidad, vectores unitarios $a_{1} y a_{2}$
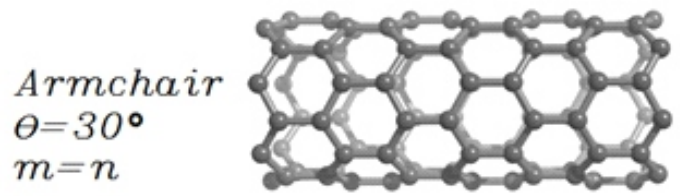

Zigzag

$\theta=0^{\circ}$

$n=0$

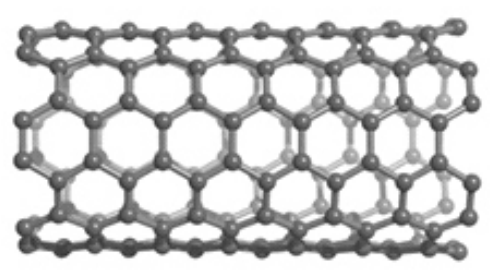

\section{Quiral \\ $0^{\circ}<\theta<30^{\circ}$ \\ $m \neq n$}

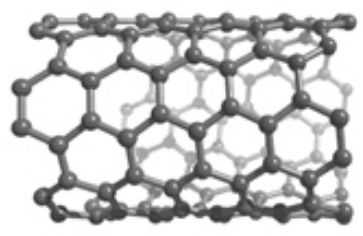

Figura 3. Clasificación de los CNTs por la quiralidad. computacional, respectivamente. Entonces, es necesario desarrollar otras formas de análisis para identificar las propiedades y comportamiento de los nanotubos de carbono, que permitan obtener resultados de forma rápida y con un bajo costo computacional, Maiti (2008). Inicialmente, se propusieron métodos teóricos basados en la mecánica clásica, Odegard et al. (2002), los cuales impiden realizar una caracterización atómica y una visualización de comportamientos no considerados por la mecánica tradicional relacionados con las fuerzas interatómicas.

Por lo tanto, otros investigadores proponen enlazar la química computacional con la mecánica de sólidos reemplazando estructuras moleculares discretas con modelos continuos equivalentes. $\mathrm{Li}$ y Chou (2003 a, b) propusieron un método analítico tipo multiescala denominado mecánica molecular estructural. Ellos, considerando la semejanza entre los fullerenos y las estructuras geodésicas, y basados en el modelo analítico mecánico para estas estructuras (tipo armadura), plantearon un acople entre energías de deformación atómicas y macroscópicas para modelar los nanotubos de carbono. Esta y otras técnicas similares han sido desarrolladas para identificar las propiedades y el comportamiento mecánico de los nanotubos de carbono Fan et al. (2009), Giannopoulos et al. (2008), Sakhaee-Pour et al. (2008), Tserpes y Papanikos (2005).

Teniendo en cuenta las consideraciones anteriores, en este trabajo se usa el método de la mecánica molecular estructural con el propósito de calcular el módulo de elasticidad y la frecuencia natural de nanotubos de carbono considerando diferentes valores en los parámetros de quiralidad, diámetro y longitud. Los resultados obtenidos permiten validar este método para estudiar las propiedades mecánicas de los CNTs y muestran que la quiralidad, el diámetro y longitud son parámetros que se deben considerar en el diseño estructural de los nanodispositivos, nanosensores y nanomáquinas.

Este artículo se organiza de la siguiente manera: en la segunda sección se presenta el método de la mecánica molecular estructural considerando los balances de energías moleculares y macroscópicas, y el enlace entre ellas; también se 
describe el Modelamiento de los nanotubos de carbono mediante el método de elementos finitos (FEM, "Finite Elements Method"). En la tercera sección se presentan los resultados de simulación del cálculo del módulo de Young y las frecuencias naturales de nanotubos de carbono con diferentes quiralidades, diámetros y longitudes. Finalmente, en la cuarta sección se presentan las conclusiones de este trabajo.

\section{Modelamiento de nanotubos de carbono usando el método la mecánica molecular estructural}

En esta sección se presenta el modelamiento de los enlaces moleculares mediante balances de energía según la mecánica molecular y la mecánica del medio continuo. También se describe el modelamiento de los nanotubos de carbono usando elementos finitos en ANSYS.

\subsection{Modelamiento de los enlaces moleculares}

El método multiescala propuesto por Li y Chou (2003 a, b), consiste en modelar la estructura del nanotubo asumiendo los enlaces covalentes de los átomos de carbonos como vigas y las uniones (nodos) como los átomos con la masa concentrada, en este modelo el radio de los átomos se desprecia por ser muy pequeño en comparación con la longitud de los enlaces. De esta forma, se genera una matriz de rigidez, donde las propiedades de las vigas se modelan haciendo una equivalencia de energías entre la mecánica molecular y la energía de deformación elástica de una viga considerando la mecánica clásica.

\subsubsection{Balance de energía estérica de la mecánica molecular}

En la Figura 4 se muestran las interacciones atómicas para calcular la energía potencial de un nanosistema (llamada energía estérica) en función de las posiciones de los átomos según la mecánica molecular, la cual considera como parámetros las constantes de las fuerzas de tensión y flexión del enlace, y permite interacciones entre átomos no enlazados. Un campo de fuerza se expresa como la energía potencial estérica y se describe en la Ec. (1).

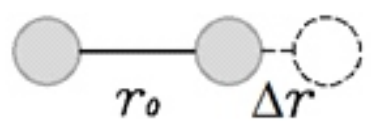

Estiramiento

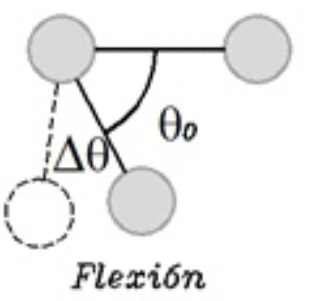

Flexion

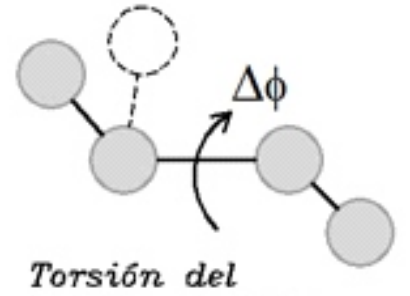

angulo diedrico

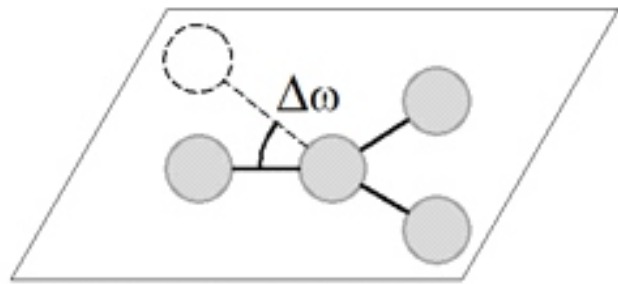

Torsion fuera del plano

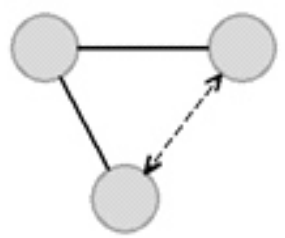

Van der Walls

Figura 4. Interacciones atómicas debidas a los enlaces y no enlaces. 
En donde Ur es la energía de estiramiento, U $\theta$ es la energía de flexión, UØ es la energía debida al ángulo de torsión dihédrico, Uw la energía de torsión fuera del plano y Uvdw la energía debida a las fuerzas de atracciónrepulsión de van der Waals.

$$
U_{\text {total }}=\Sigma U_{r}+\Sigma U_{\theta}+\Sigma U_{\varnothing}+\Sigma U_{\omega}+\Sigma U_{v d w}
$$

Las interacciones de van der Waals son despreciables en comparación a las fuerzas ejercidas por los enlaces covalentes entre los átomos de carbono. De igual forma, para deformaciones pequeñas se utiliza una aproximación armónica que permite que las energías debidas a la torsión fuera del plano y dihédrica se consideren en un solo término. Los valores de las energías de los tres términos resultantes están dados por las Ec. (2), (3) y (4).

$$
\begin{gathered}
U_{r}=1 / 2 \mathrm{k}_{r}\left(r-r_{0}\right)^{2} 1 / 2 k_{r}(\Delta r)^{2} \\
U_{\theta}=1 / 2 \mathrm{k}_{\theta}\left(r-r_{0}\right)^{2} 1 / 2 k_{\theta}(\Delta \theta)^{2} \\
U_{\tau}=U_{\varnothing}+U_{\omega}=1 / 2 k_{\tau}(\Delta \emptyset)^{2}
\end{gathered}
$$

En donde $k_{r} k_{\theta}$ y $k_{\tau}$ son las constantes de: estiramiento de enlace, resistencia a la flexión y resistencia a la torsión; $\Delta r, \Delta \theta$ y $\Delta \varnothing$ representan el incremento en el estiramiento del enlace, la variación del ángulo de enlace y la variación del ángulo de enlace por torsión, respectivamente.

\subsubsection{Balance de la energía de deformación de la mecánica del medio continuo}

Para modelar la energía de deformación de una viga elástica de área transversal constante se consideran los cambios en la energía debidos a fuerzas axiales UA, momentos flectores UM y momentos torsores UT descritos por las Ec. (5), (6) y (7).

$$
\begin{aligned}
& U_{A}=1 / 2 \int_{0}^{L} \frac{N^{2}}{E A} d L=1 / 2 \frac{L N^{2}}{E A}=1 / 2 \frac{E A}{L}(\Delta L)^{2} \\
& U_{M}=1 / 2 \int_{0}^{L} \frac{M^{2}}{E I} d L=1 / 2 \frac{E I}{L} a^{2}=1 / 2 \frac{E I}{L}(2 a)^{2} \\
& U_{T}=1 / 2 \int_{0}^{L} \frac{T^{2}}{G J} d L=1 / 2 \frac{L T^{2}}{G J}=1 / 2 \frac{G J}{L}(\Delta \beta)^{2}
\end{aligned}
$$

En donde $\mathrm{N}$ es la fuerza normal, $\mathrm{M}$ un momento flector, T un par torsor, E el módulo de elasticidad, A el área transversal, L la longitud, G el modulo de cortante, $\mathrm{J}$ el momento polar de inercia, $\Delta \mathrm{L}$ la elongación, $\Delta \alpha$ el ángulo de rotación en los extremos de la viga y $\Delta \beta$ la rotación relativa entre los extremos de la viga.

\subsubsection{Enlace de energías para determinar las constantes en el modelo de viga elástica}

Considerando enlaces entre las energías de la mecánica molecular y la mecánica del medio continuo, se obtienen las Ec. (8), (9) y (10). Se asume que el área transversal de la viga es circular, con diámetro d, área $A=\pi d^{2} / 4$, momento de inercia $I=\pi d^{4} / 64$, momento polar de inercia $J=\pi d^{4} / 32$, y longitud $\mathrm{L}=0.1421 \mathrm{~nm}$, que es la distancia del enlace doble entre átomos de carbono en un sistema en equilibrio $\left(\mathrm{a}_{c-c}\right)$.

$$
\begin{aligned}
& \frac{E A}{L}=k_{r} \\
& \frac{E I}{L}=k_{\bullet} \\
& \frac{G J}{L}=k_{\tau}
\end{aligned}
$$

Donde las constantes moleculares para el carbono se obtienen de estudios basados sobre campos de fuerza, Cornell et al. (1995); $\mathrm{kr}=938 \mathrm{kcal} \mathrm{mol}^{-1} \AA^{-2}$ $=6.52 \times 10^{-7} \mathrm{~N} \mathrm{~nm}^{-1}, \mathrm{k}_{\theta}=126 \mathrm{kcal} \mathrm{mol}^{-1} \mathrm{rad}^{-2}=$ $8.76 \times 10^{-10} \mathrm{~N} \mathrm{~nm} \mathrm{rad}^{-2}, \mathrm{k}_{\tau}=40 \mathrm{kcal} \mathrm{mol}^{-1} \mathrm{rad}^{-2}=2.78$ $\mathrm{x} 10^{-10} \mathrm{~N} \mathrm{~nm} \mathrm{rad}^{-2}$.

Reemplazando los valores anteriores en las Ec. (8), (9) y (10) se obtienen $\mathrm{L}=0.1421 \mathrm{~nm}$, $\mathrm{d}=0.147 \mathrm{~nm}, \mathrm{E}=5.49 \mathrm{TPa}$ y $\mathrm{G}=0.871 \mathrm{TPa}$ para cada elemento tipo viga. El radio del átomo $\mathrm{r}_{\mathrm{c}}=2.7 \times 10^{-5}$ $\AA$ es despreciable, y la masa se asume como puntual en los nodos (átomos de carbono) y tiene un valor de $\mathrm{m}_{\mathrm{c}}=1.9943 \times 10^{-23} \mathrm{~g}$. 


\subsection{Modelamiento de los nanotubos de carbono usando elementos finitos}

Los nanotubos de carbono se modelan en elementos finitos usando el programa ANSYS 12. Con el propósito de mitigar los errores numéricos por underflow, y considerando que las longitudes $\mathrm{y}$ masas son muy pequeñas, se utilizan los siguientes ajustes de normalización:

$$
L_{a n}=10^{10} L, F_{a n}=10^{20} F, M_{a n}=10^{26} M
$$

Donde el subíndice an es el valor asociado en ANSYS a las dimensiones reales de longitud L en $\mathrm{m}$, fuerza $\mathrm{F}$ en $\mathrm{N}$ y masa $\mathrm{M}$ en $\mathrm{kg}$. Esta normalización ocasiona ajustes en E: el módulo de elasticidad en Pa; G: el módulo de cortante en Pa; y f: la frecuencia en $\mathrm{Hz}$.

$$
E_{a n}=E, G_{a n}=G, f_{a n}=10^{-8} f,
$$

Para los enlaces entre átomos de carbono se usa el elemento uniaxial tipo viga BEAM4 que permite llevar a cabo análisis en tensión, compresión, torsión y pandeo; este elemento está definido por 2 o 3 nodos según la orientación de la viga y tiene 6 grados de libertad por cada uno de ellos. Los requerimientos son el área transversal, 2 momentos de inercia, 2 direcciones y las propiedades del material. Para determinar los modos de vibración del nanotubo se ubican las masas puntuales en los extremos de las vigas con el elemento puntual de masa MASS21, que tiene 6 grados de libertad y concentra los componentes de masa e inercia en un solo punto, Ansys Inc (2009).

La geometría se modela utilizando APDL de ANSYS para generar los puntos y uniones de nanotubos de tipo armchair y zigzag de cualquier longitud.

\section{Resultados de simulación, cálculos y análisis del módulo de elasticidad y las frecuencias de vibración de nanotubos de carbono}

En esta sección se presentan los resultados de simulación y cálculos para el módulo de elasticidad y las frecuencias naturales de los nanotubos de carbono para diferentes quiralidades, diámetros y longitudes. También se presenta un análisis detallado sobre los resultados obtenidos.

\subsection{Módulo de elasticidad de nanotubos de carbono}

Para determinar el módulo de elasticidad de los nanotubos de carbono se usa un modelo estático, en el que se calcula la elongación total $\mathrm{HN}$ como el resultado de las interacciones entre los enlaces. El módulo de elasticidad está dado por la Ec. (11) y el subíndice $\mathrm{N}$ índica que son dimensiones del nanotubo.

$$
E_{N}=\frac{F_{N} / A_{N}}{H_{N} / H_{N o}}
$$

Donde FN es la fuerza total aplicada y AN el área transversal según la Ec. (12).

$$
A_{N}=\pi \mathrm{d}_{N} \mathrm{t}
$$

Donde $\mathrm{dN}$ es el diámetro del nanotubo según la quiralidad y t el espesor efectivo de capa. dN está descrito por la Ec. (13)

$$
d_{N}=\sqrt{3\left(n^{2}+m^{2}+n m\right)} a_{c-c} / \pi
$$

En la literatura se han propuesto diferentes espesores efectivos de capa para diferentes métodos teóricos, que van desde 0.064 hasta 0.69 $\mathrm{nm}$, pero la mayoría de estudios proponen un espesor de capa igual a $0.34 \mathrm{~nm}$ que es la distancia de separación entre capas de MWCNTs. En la Tabla 1 se presentan los valores calculados en este trabajo para un nanotubo de carbono con quiralidad $(8,8)$ y diferentes métodos de análisis con diferentes espesores de capa.

Los resultados obtenidos son acordes a los reportados en otros estudios que usaron métodos cuánticos, moleculares y multiescala, lo cual valida el procedimiento estático del método de la mecánica molecular estructural. La mayoría de los resultados reportados presentan datos generales del módulo de elasticidad sin especificar la quiralidad, el diámetro y la longitud del nanotubo, sin embargo, estos parámetros deben ser considerados para el modelamiento y el diseño. En 
Tabla 1. Módulo de elasticidad para diferentes espesores propuestos de un nanotubo de carbono $(8,8)$

\begin{tabular}{|c|c|c|c|c|}
\hline Investigadores & Método & $\begin{array}{l}\text { Espesor de } \\
\text { capa }[\mathrm{nm}]\end{array}$ & $\begin{array}{c}E \\
{[T P a]}\end{array}$ & $\begin{array}{c}\text { E trabajo } \\
\text { actual } \\
\text { [TPa] }\end{array}$ \\
\hline Tombler et al. (2000) & Experimental: 3 puntos de flexión & & 1.2 & \\
\hline Cooper et al. (2001) & Experimental: Espectroscopia Raman & & $0.78-2.34$ & \\
\hline Lourie, Wagner (1998) & Experimental: Espectroscopia Raman & & 2.8-3.6 & \\
\hline Yacobson et al (1996) & Dinámica Molecular & 0.066 & 5.5 & 5.36 \\
\hline Xin et al (2000) & Teoría de banda de energía electrónica & 0.074 & 5.1 & 4.78 \\
\hline Pantano et al.(2004) & Mecánica clásica, capas continuas & 0.075 & 4.84 & 4.71 \\
\hline Tu et al. (2002) & Modelo aproximado de densidad local & 0.075 & 4.7 & 4.71 \\
\hline Kudin et al (2001) & ab initio & 0.0894 & 3.86 & 3.95 \\
\hline Tserpes, Papanikos (2005) & Multiescala: Dinámica molecular+FEM & 0.147 & 2.38 & 2.40 \\
\hline Hernández et al (1998) & $\begin{array}{c}\text { Dinámica molecular: orbitales moleculares(Teoría } \\
\text { de funcionales de la densidad DFT) }\end{array}$ & 0.34 & 1.24 & 1.04 \\
\hline Jin, Yuan (2003) & Dinámica molecular & 0.34 & 1.238 & 1.04 \\
\hline Li, Chou (2003a) & $\begin{array}{l}\text { Multiescala: campos de fuerzas moleculares }+ \\
\text { matriz de rigidez }\end{array}$ & 0.34 & 1.02 & 1.04 \\
\hline Sears, Batra (2004) & Mecánica molecular & 0.34 & 0.99 & 1.04 \\
\hline Lu (1997) & $\begin{array}{l}\text { Dinámica molecular (modelo empírico de } \\
\text { constantes de fuerza }\end{array}$ & 0.34 & 0.975 & 1.04 \\
\hline Odegard (2002) & Modelo continuo equivalente & 0.69 & & 0.51 \\
\hline Este Trabajo & $\begin{array}{l}\text { Multiescala: Campos de fuerzas } \\
\text { moleculares+FEM }\end{array}$ & 0.34 & & 1.04 \\
\hline
\end{tabular}

la Figura 5 se presentan las variaciones del módulo de Young, el cual es inversamente proporcional al espesor de capa. Los valores encontrados usando el valor de $0.34 \mathrm{~nm}$ son acordes a los resultados experimentales, por esta razón se selecciona este espesor de capa para el modelamiento de los CNTs.

Teniendo en cuenta lo anterior, los parámetros a evaluar son la quiralidad y el diámetro del nanotubo para el módulo de elasticidad. Simulando una prueba de tensión (ver Figura 6a) en nanotubos tipo armchair y zigzag de diferentes diámetros, se calcula el módulo de elasticidad para los CNTs con relaciones de longitud / radio $(\mathrm{LN} / \mathrm{rN})$ entre 10.20 y 10.45 . Estos resultados son mostrados en la Figura 7 y el comportamiento de la curva es similar al presentado en otros estudios que realizan análisis teóricos, como en Thostenson et al. (2001). Adicionalmente se observa una mayor dependencia del módulo de elasticidad con respecto al diámetro que a la quiralidad, y el módulo se incrementa en forma no lineal con respecto al diámetro; también, el módulo es mayor en los nanotubos tipo armchair que en los zigzag, esta diferencia es más acentuada para los diámetros más grandes. 


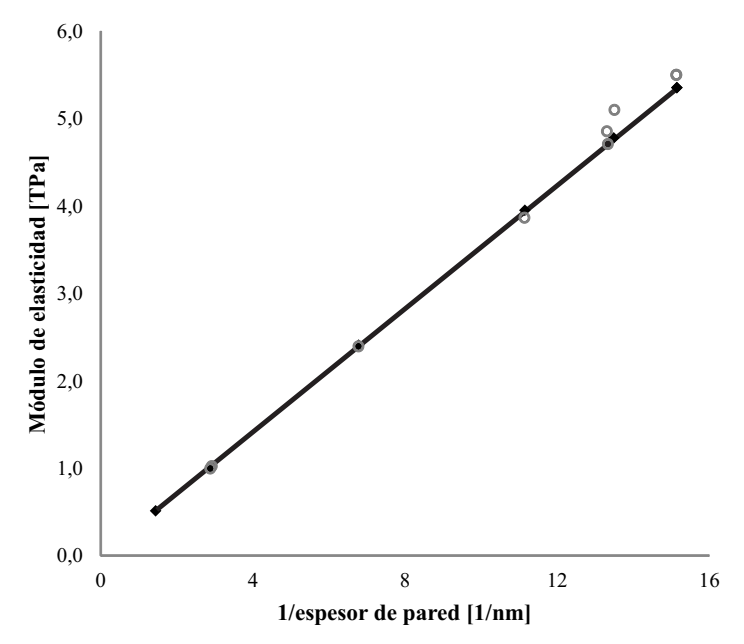

Figura5. Variación del módulo de elasticidad de un nanotubo de carbono $(8,8)$ con diferentes espesores de capa.

- Este trabajo, o Otros trabajos.

$a$

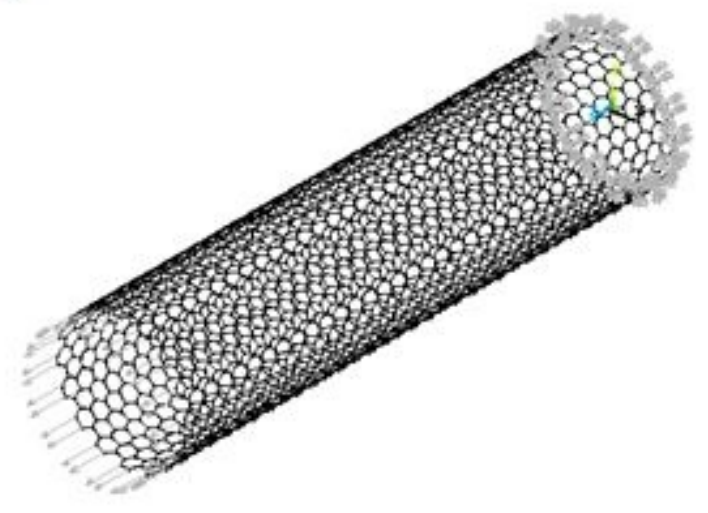

b.

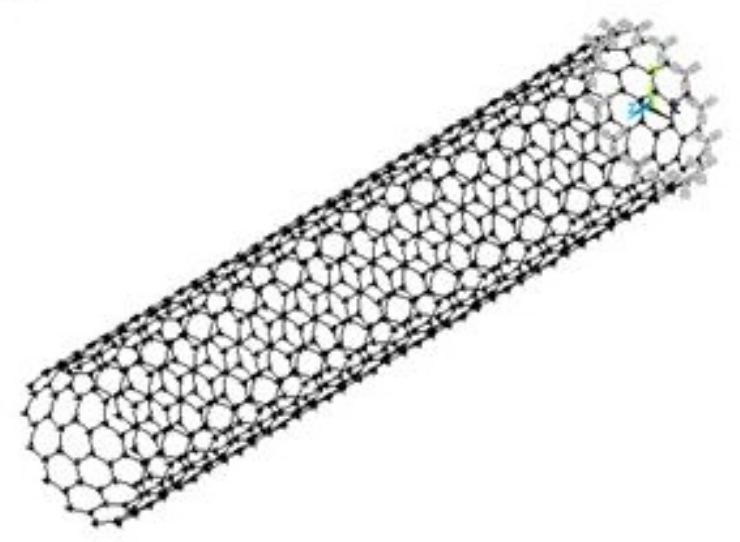

Figura 6. Nanotubos de carbono con fuerzas aplicadas: a.armchair sometido a tensión, b. zigzag empotrado con las masas puntuales en los nodos.
Considerando que la elongación total del nanotubo es el resultado de la interacción entre los enlaces, una posible explicación de la diferencia del valor para el módulo de elasticidad entre nanotubos tipo armchair y zigzag es la dirección de los enlaces con respecto a la dirección de la carga. En el nanotubo tipo armchair todos los enlaces forman ángulos con respecto a la dirección de la fuerza, mientras que en los zigzag un tercio de los enlaces están alineados con ella.

Algunos autores, Li y Chou (2003a) y Tserpes y Papanikos (2005), proponen que la dependencia del módulo de elasticidad con respecto al diámetro se debe a la curvatura del nanotubo. Entre más pequeño sea el diámetro, el efecto de la curvatura genera una mayor distorsión de los enlaces carbono-carbono de la red cristalina, mientras que si el diámetro aumenta la curvatura disminuye gradualmente aproximándose a una capa de grafeno, por lo cual el módulo de elasticidad alcanza aproximadamente el mismo valor de éste, 1.03-1.06 TPa, Han et al. (2010). Este efecto se puede observar en la Figura 8, la cual muestra nanotubos tipo armchair $(6,6),(10,10)$ y $(12,12)$; a medida que el diámetro aumenta, los enlaces forman ángulos cada vez más pequeños con los enlaces vecinos, generando una mayor estabilidad de la estructura. Entonces, el comportamiento elástico de los nanotubos de carbono es consecuencia directa de su estructura atómica, debido tanto a la quiralidad, como a la curvatura del diámetro.

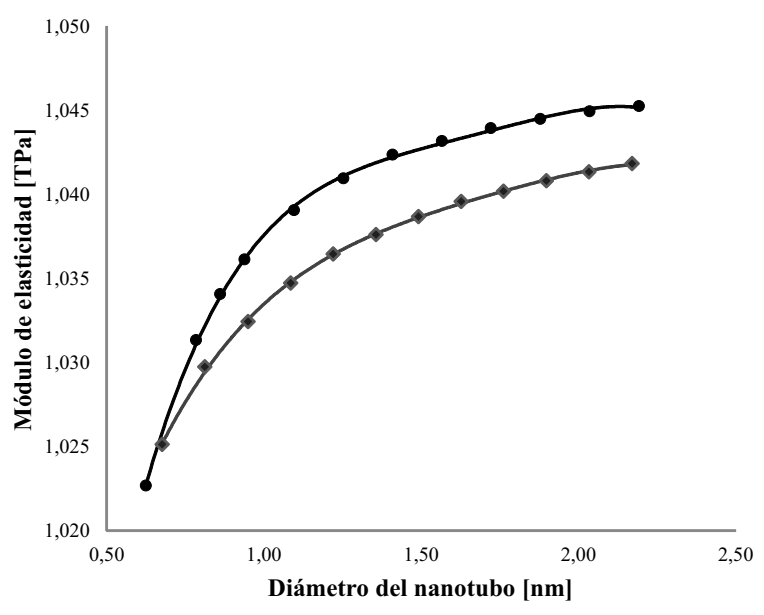

Figura 7. Módulo de elasticidad de nanotubo de carbono de diferentes diámetros y quiralidades. armchair, $\bullet$ zigzag. 

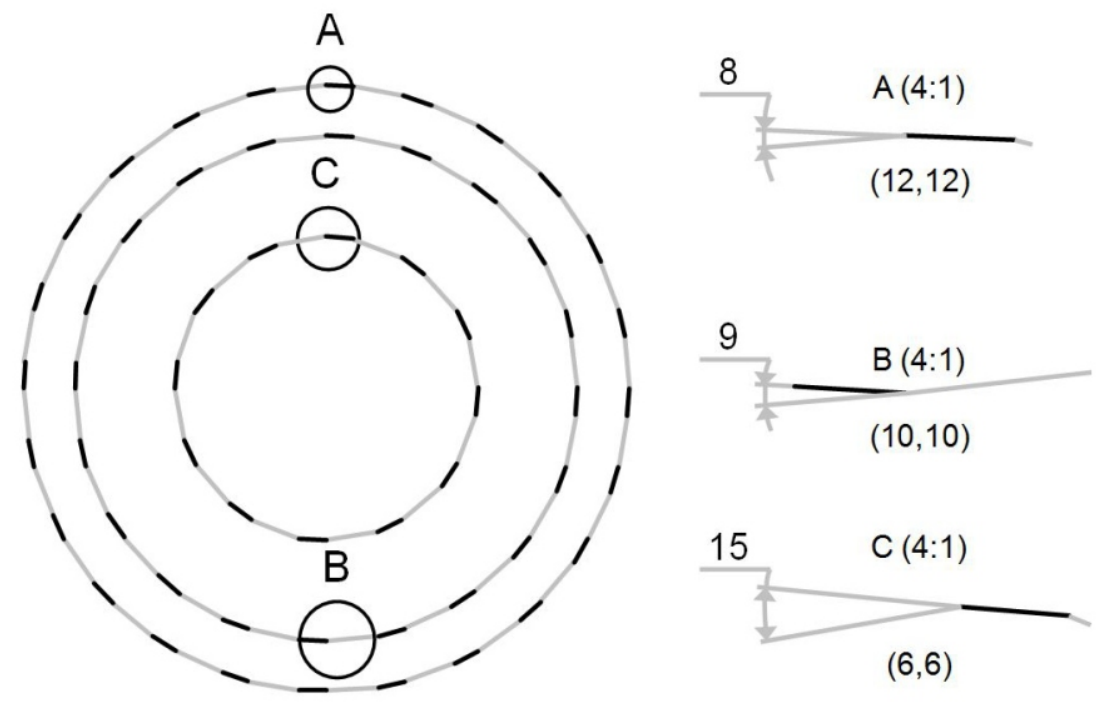

$(6,6)$

Figura 8. Curvatura por los enlaces en nanotubos $(6,6),(10,10)$ y $(12,12)$

Como segundo parámetro se estudió la dependencia de la longitud del nanotubo en el módulo de elasticidad, en este caso se realizaron simulaciones para tres nanotubos tipo armchair y tipo zigzag con relaciones de longitud/radio entre 10 y 70. En la Figura 9 se presentan estos resultados.

Las curvas del módulo de elasticidad con respecto a la relación longitud/radio de los nanotubos de carbono presentan un comportamiento no lineal.
Para los nanotubos tipo zigzag la variación es despreciable, pero para los nanotubos tipo armchair se observa una mayor dependencia de la longitud, especialmente cuando el diámetro es pequeño; por ejemplo para el caso $(6,6)$ considerando el cambio en la relación $\mathrm{L} / \mathrm{r}$ entre 10 y 30, el módulo de elasticidad presenta una diferencia mayor a 10GPa. En todos los nanotubos se observa que para relaciones $\mathrm{L} / \mathrm{r}$ mayores a 30 , el módulo tiende a estabilizarse y las curvas convergen asintóticamente.
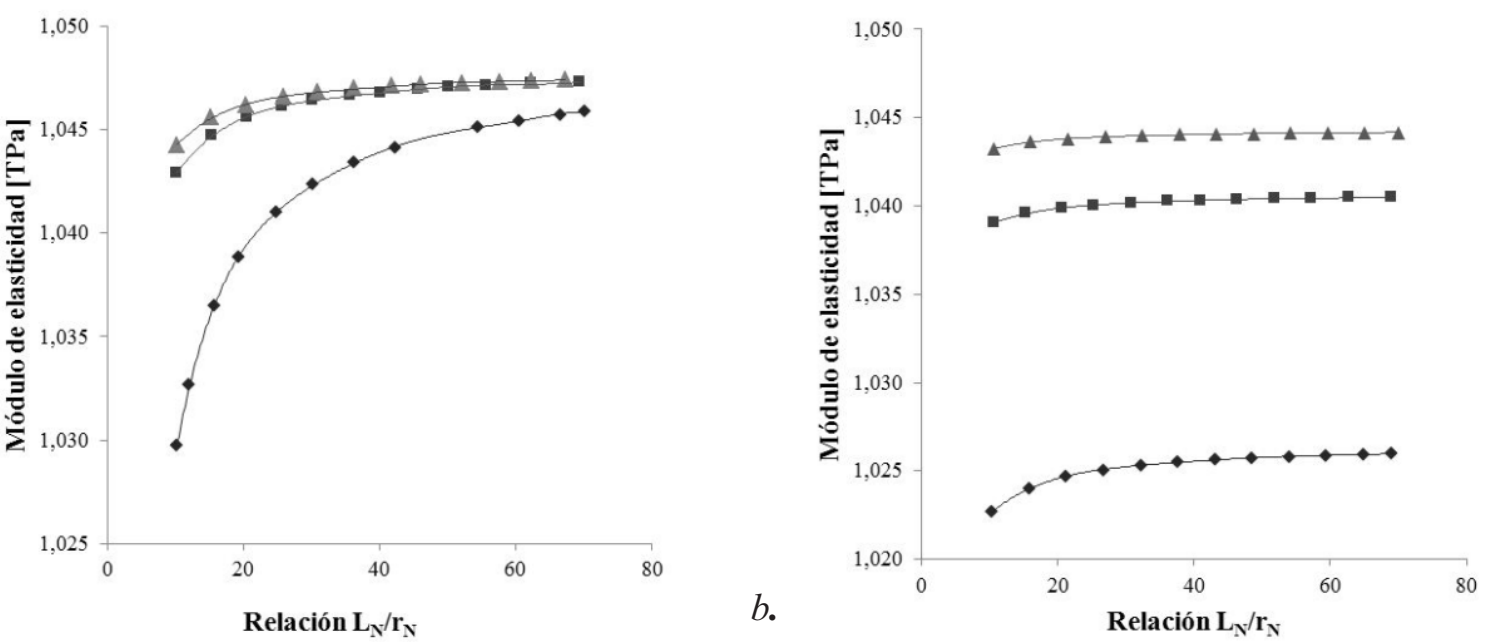

Figura 9. Módulo de elasticidad según relación longitud/radio para nanotubos a armchair, $(6,6)$ $\boldsymbol{\Delta}(16,16)$ b.zigzag $(8,0) \mathbf{\square}(14,0) \boldsymbol{\Delta}(20,0)$. 
Los nanotubos de diámetros más pequeños son los que presentan una mayor variación en la curva del módulo de elasticidad vs longitud/radio, esto se debe al efecto de la distorsión de los enlaces por la curvatura como se mencionó anteriormente. El efecto de la quiralidad se muestra en la Figura 10 para las estructuras $(6,6)$ y $(8,0)$, cada una con 3 hileras de celdas hexagonales a lo largo del eje. Se puede observar que longitudinalmente el nanotubo armchair tiene un número irregular de átomos ( 3 o 4$)$, mientras que en el zigzag el número de átomos es constante (4); la anterior irregularidad y la cantidad de enlaces paralelos a la fuerza, ocasionan una variación en la elongación respecto a la longitud inicial. Cuando la longitud es mayor (mayor cantidad de celdas hexagonales en la dirección del eje), la relación de deformación del nanotubo se estabiliza puesto que la diferencia en la cantidad de átomos se hace menos significativa; dando como resultado la convergencia del módulo de elasticidad observado en la Figura 9.

\subsection{Frecuencias naturales de nanotubos de carbono}

Con el propósito de validar el método multiescala de la mecánica molecular estructural para los análisis modales, se llevaron a cabo simulaciones para un nanotubo $(5,5)$ con diferentes relaciones de longitud-radio, y los resultados se compararon con los obtenidos en dinámica molecular, Duan et al. (2007) y mecánica molecular, Chowdhury et al. (2010). Los resultados de simulación de la primera frecuencia natural en $\mathrm{THz}$ para los tres métodos se muestran en la Tabla 2, y se obtuvieron diferencias aceptables, menores al 7\%. Además, en las q.

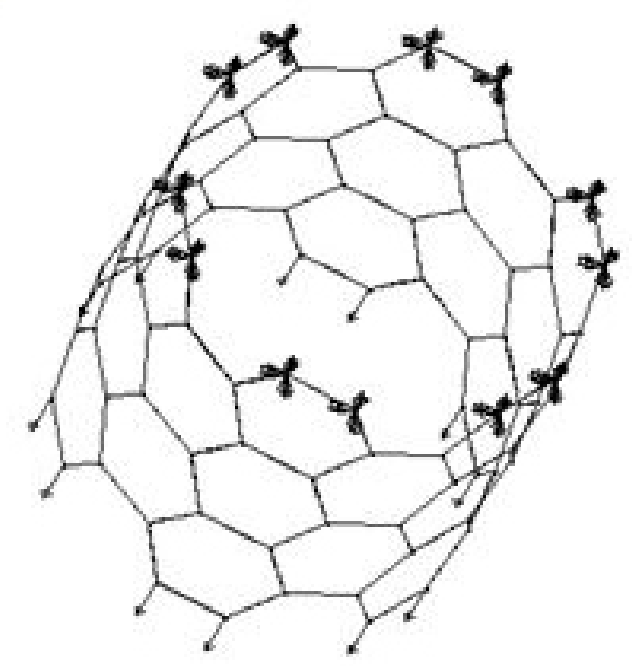

b.

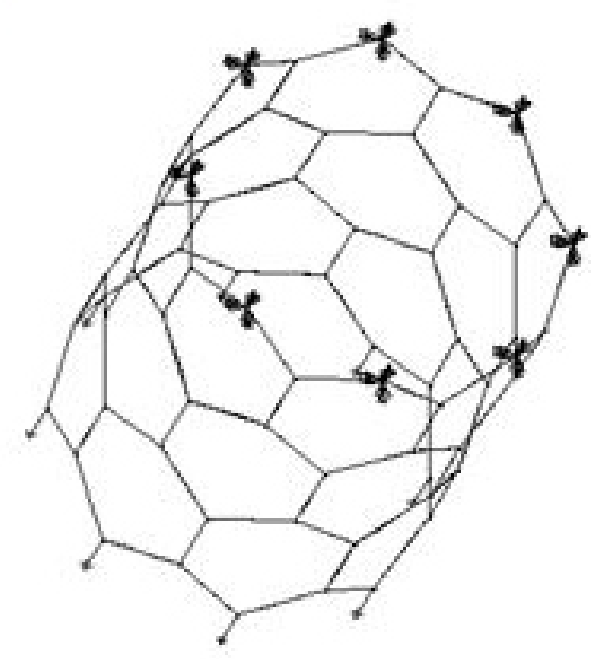

Figura 10. Estructura de nanotubo a. armchair $(6,6)$ y b. zigzag $(8,0)$

Tabla 2. Comparación de resultados par la primera frecuencia natural en $\mathrm{GHz}$, del método multiescala empleado con dinámica y mecánica molecular

\begin{tabular}{cccccc}
\hline & $\begin{array}{c}\text { Trabajo actual } \\
\text { [Tpa] }\end{array}$ & $\begin{array}{c}\text { Mecánica } \\
\text { molecular } \\
{[\text { TPa] }}\end{array}$ & Diferencia & $\begin{array}{c}\text { Dinámica } \\
\text { molecular } \\
{[\text { TPa] }}\end{array}$ & Diferencia \\
10.52 & 0.211 & 0.220 & $4.3 \%$ & 0.212 & $0.5 \%$ \\
12.70 & 0.148 & 0.156 & $5.5 \%$ & 0.150 & $1.4 \%$ \\
14.15 & 0.120 & 0.128 & $6.4 \%$ & 0.123 & $2.3 \%$ \\
16.32 & 0.092 & 0.098 & $6.3 \%$ & 0.094 & $2.0 \%$ \\
\hline
\end{tabular}


simulaciones realizadas con los métodos de la mecánica molecular estructural y de la mecánica molecular, el valor de los dos primeros modos de vibración es el mismo, pero con diferentes planos de simetría. Entonces, los resultados confirman la validez de utilizar el método multiescala en el análisis modal de los nanotubos.

Mediante técnicas experimentales, como espectroscopía Raman y difracción de rayos X se han observado posibles comportamientos vibratorios de los nanotubos de carbono, Gao et al. (1998), Li y Chang (2009), sin embargo, por las limitaciones de las pruebas experimentales los resultados no son comparables con los obtenidos en las simulaciones ya que no consideran parámetros como diámetro, quiralidad y longitud de los nanotubos simulados, además las condiciones de borde empleadas son diferentes. No obstante, los resultados de simulación y los experimentales concuerdan en que las frecuencias para los CNTs están en la escala de los GHz, y con valores mayores a los de los nanoresonadores de alta frecuencia como los fabricados con carburo de silicio (SiC), Jiang et al. (2006). Ésta característica es aprovechada para diseñar sensores de masa debido a su alta resolución y velocidad de la señal.

Una vez validado el método multiescala para análisis modal y con el propósito de evaluar el efecto de la quiralidad y la relación longitud/radio sobre las frecuencias naturales del sistema, se simulan los nanotubos $(6,6),(12,12),(16,16)$, $(8,0),(14,0)$ y $(20,0)$ con diferentes longitudes y en configuración de viga en voladizo. Estos resultados se presentan en la Figura 11, en la cual se muestran las frecuencias naturales de nanotubos armchair y zigzag para relaciones $\mathrm{LN} / \mathrm{rN}$ entre 10 y 70.

El comportamiento de las curvas de las frecuencias mostradas en la Figura 11, es acorde con los resultados presentados por otros autores, Zhao et al. (2002), Li y Chou (2003b), Chowdhury et al. (2010) y Duan et al. (2007). En ésta Figura se observa que las curvas presentan un comportamiento no lineal con respecto a la relación $\mathrm{LN} / \mathrm{rN}$, de modo que la frecuencia natural disminuye con el aumento del diámetro o la longitud. También se observó que el efecto de la
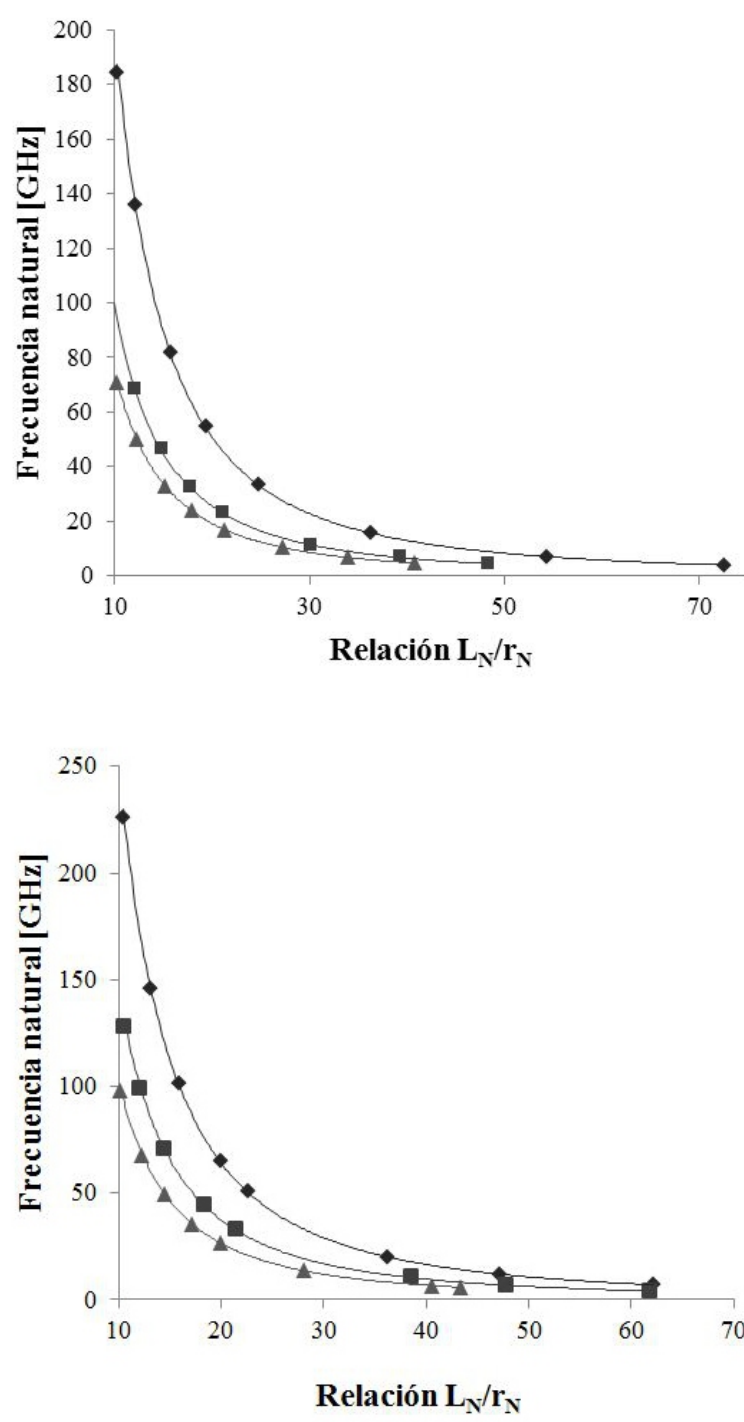

Figura 11. Modos de vibración según la relación longitud/radio para nanotubos a. armchair, $(6,6)$ $\mathbf{u}(12,12) \boldsymbol{\Delta}(16,16)$ b. zigzag, $\bullet(8,0) \boldsymbol{\square}(14,0)$ $\mathbf{\Delta}(20,0)$.

quiralidad no es significativo, por ejemplo para diámetros similares como los presentados en los CNTs $(20,0)$ y $(12,12)$ (diámetros de $1.56 \mathrm{~nm}$ y $1.63 \mathrm{~nm}$, respectivamente) los valores de las frecuencias son cercanos.

Con el fin de comparar el comportamiento entre los CNTs como vigas en voladizo y una viga de material continuo, se realizó una linealización para las curvas de frecuencia y se obtuvo el 
modelo matemático descrito por la Ec. (14), en donde $\omega \mathrm{nN}$ es la frecuencia natural del nanotubo; los valores del coeficiente a varían con la quiralidad y el diámetro del nanotubo.

Teniendo en cuenta los resultados obtenidos de la linealización se calcularon los valores de los coeficientes para cada quiralidad, lo cual se muestra en la Tabla 3. Al observar los coeficientes de los nanotubos $(20,0)$ y $(12,12)$, se confirma que el efecto de la quiralidad en los valores de la frecuencia natural es irrelevante, y el coeficiente a decrece cuando aumenta el diámetro, pero no de forma proporcional.

Tabla 3. Coeficientes de la ecuación de las curvas $\omega_{N} v s L_{N} / r_{N}$

\begin{tabular}{cc}
\hline Nanotubo & Coeficiente $a$ \\
$(6,6)$ & $1.82 \times 10^{4}$ \\
$(12,12)$ & $9.10 \times 10^{3}$ \\
$(16,16)$ & $6.82 \times 10^{3}$ \\
$(8,0)$ & $2.27 \times 10^{4}$ \\
$(14,0)$ & $1.34 \times 10^{4}$ \\
$(20,0)$ & $9.37 \times 10^{3}$ \\
\hline
\end{tabular}

Los resultados obtenidos están de acuerdo con la teoría para vigas elásticas en voladizo y de área transversal constante, cuyo comportamiento está dado por la Ec. (15), donde $\omega n$ es la frecuencia natural de la viga continua, $\beta i$ es la constante del modo i según las condiciones de borde, $\mathrm{E}$ es el módulo de elasticidad y es constante para un material convencional, I es el momento de inercia y es constante para un área transversal constante, $q$ es la distribución lineal de masa o masa por unidad de longitud y L es la longitud de la viga.

Para una viga de material continuo q es constante y la longitud $\mathrm{L}$ afecta la frecuencia natural por un factor de $\mathrm{L}^{-2}$. Sin embargo, en el nanotubo de carbono la masa no es continua sino puntual en los nodos de la estructura, lo cual implica que el comportamiento de la frecuencia se afecta con el cambio de longitud de forma $\mathrm{L}^{-1.96}$.

$$
\begin{gathered}
\omega_{n N}=a\left(\frac{L_{N}}{r_{N}}\right)^{1.96} \\
\omega_{n}=\beta_{i}^{2} \sqrt{\frac{E I}{q L^{4}}}
\end{gathered}
$$

En el análisis modal realizado, se obtuvieron los diez primeros modos de vibración para nanotubos armchair y zigzag en configuración de cantiléver, los cuales se muestran en la Figura 12. Estos modos son similares a los reportados en estudios que usan métodos multiescala, sin embargo, en la literatura consultada no se han reportado los modos de vibración con análisis teóricos moleculares ni experimentales, lo cual no permite la validación de los mismos.

\section{Conclusiones}

El comportamiento elástico y las frecuencias de vibración de los CNTs son consecuencia directa de su estructura atómica, es decir de los parámetros: espesor de capa, diámetro, quiralidad y relación $\mathrm{LN} / \mathrm{rN}$.

El valor del módulo de elasticidad es inversamente proporcional al espesor de capa. En este trabajo se seleccionó como espesor $0.34 \mathrm{~nm}$ que es la distancia de separación entre capas en los MWCNTs.

El módulo de elasticidad es mayor a medida que se incrementa el diámetro; con una diferencia de diámetros de $0.6 \mathrm{~nm}$ a $2.3 \mathrm{~nm}$, el módulo varía desde 1.023 TPa hasta 1.045TPa. La variación es más relevante para diámetros pequeños y el valor tiende a estabilizarse para diámetros grandes.

El módulo de elasticidad en CNTs zigzag es un poco mayor que en $\mathrm{CNTs}$ armchair para diámetros similares, la diferencia es mayor si el diámetro es grande y es de aproximadamente $5 \mathrm{GPa}$ para diámetros mayores a $1.5 \mathrm{~nm}$.

La variación de la relación $\mathrm{LN} / \mathrm{rN}$ afecta en mayor proporción el módulo de elasticidad de los nanotubos tipo armchair que de los nanotubos tipo zigzag; a partir de una relación mayor a 30 el valor del módulo de elasticidad se estabiliza tendiendo a un valor constante.

Las dos primeras frecuencias naturales de los CNTs, independientemente de la quiralidad y en configuración de cantiléver, son iguales en valor y su modo de vibración difiere en el plano de simetría. 
a.
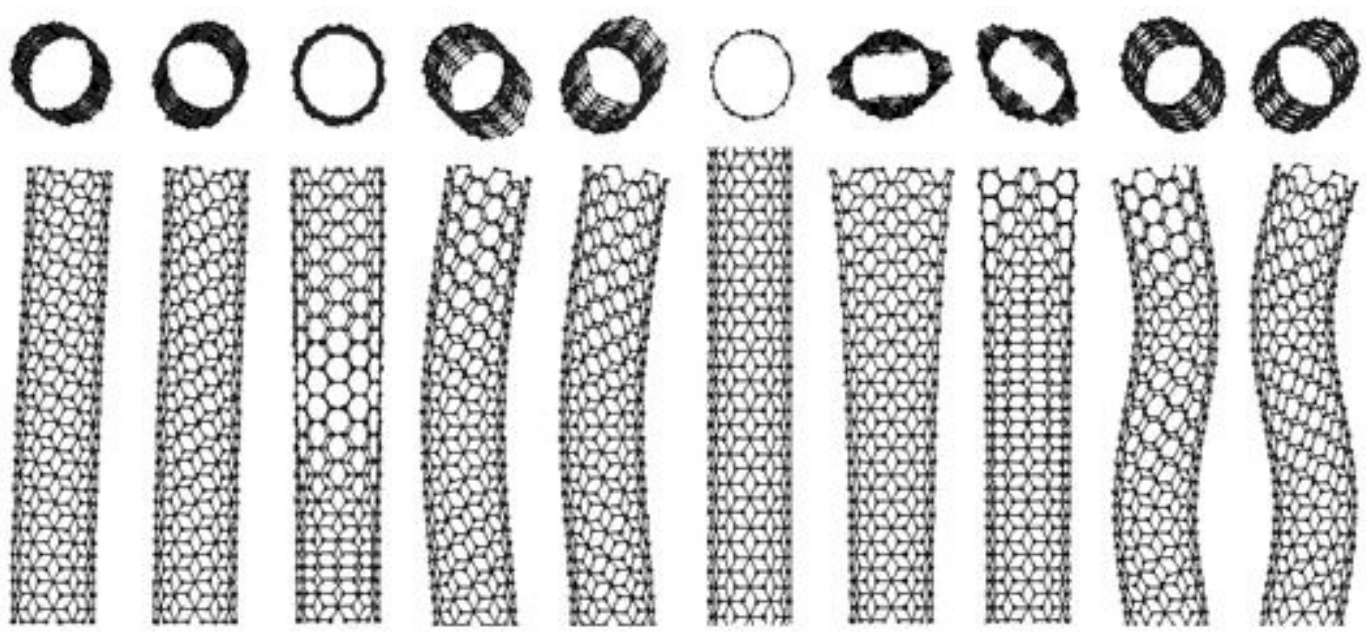

$b$.
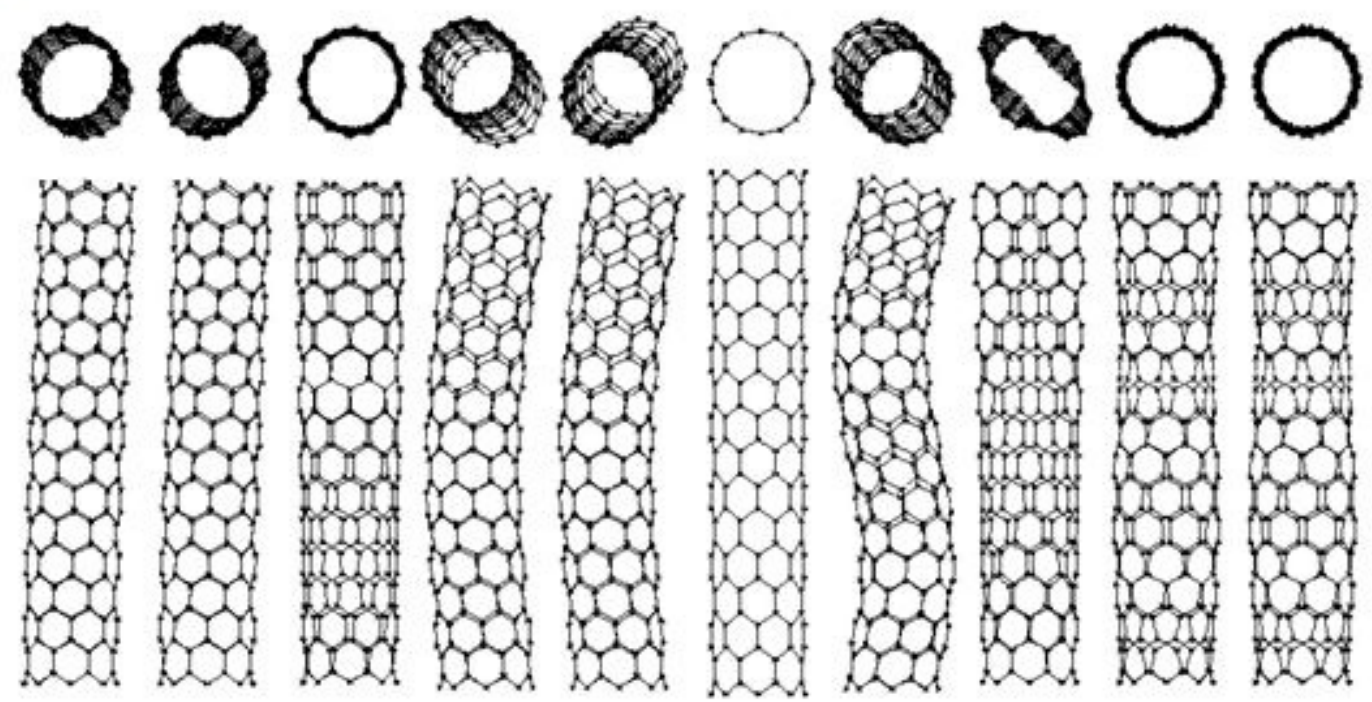

Figura 12. Modos de vibración de un nanotubo de carbono $(5,5)$ a) primer modo y b) segundo modo

Las frecuencias naturales de los nanotubos de carbono disminuyen a medida que es mayor el diámetro o la longitud, y la quiralidad no afecta estas frecuencias.

El comportamiento de las frecuencias no es lineal con respecto a la longitud y varía de la forma L1.96 según el modelo matemático desarrollado en este trabajo.

Los valores de los coeficientes para determinar las frecuencias naturales son independientes de la quiralidad, sin embargo, estos valores disminuyen cuando aumenta el diámetro del nanotubo, pero no de forma lineal.

El método multiescala de la mecánica molecular estructural permite obtener resultados confiables en algunos segundos usando pocos recursos computacionales en comparación con los métodos moleculares que tardan varias horas.

La quiralidad, el diámetro y longitud son parámetros que se deben considerar en el diseño estructural de los nanodispositivos, nanosensores y nanomáquinas. 


\section{Referencias Bibligráficas}

ANSYS, Inc. (2009) . Release Notes 12.1 Canonsburg

Chowdhury, R., Adhikari, S., Wang, C. Y. and Scarpa, F. (2010). A molecular mechanics approach for the vibration of single-walled carbon nanotubes, Computational Materials Science, 48(4), 730-735

Cooper, C. A., Young, R. J. and Halsall, M. (2001). Investigation into the deformation of carbon nanotubes and their composites through the use of Raman spectroscopy, Composites Part A-Applied Science and Manufacturing, 32(3-4), 401-411.

Cornell, W. D., Cieplak, P., Bayly, C. I., Gould, I. R., Merz, K. M., Ferguson, D. M., Spellmeyer, D. C., Fox, T., Caldwell, J. W. and Kollman, P. A. (1995). A Second Generation Force Field for the Simulation of Proteins, Nucleic Acids, and Organics Molecules, Journal of American Chemical Society, 117(19), 5179-5197.

Dresselhaus, M. S., Dresselhaus, G. and Saito, R. (1995). Physics of carbon nanotubes, Carbon, 33(7), 883-891.

Duan, W. H., Wang, C. M. and Zhang, Y. Y. (2007). Calibration of nonlocal scaling effect parameter for free vibration of carbon nanotubes by molecular dynamics, Journal of Applied Physics, 101(2).

Fan, C. W., Liu, Y. Y. and Hwu, C. (2009). Finite element simulation for estimating the mechanical properties of multi-walled carbon nanotubes, Applied Physics a-Materials Science \& Processing, 95(3), 819-831.

Gao, G., Cagin, T. and Goddard III, W. (1998). Energetics, structure, mechanical and vibrational properties of single-walled carbon nanotubes, nanotechnology, 9, 184-191.

Giannopoulos, G. I., Kakavas, P.A. and Anifantis, N. K. (2008). Evaluation of the effective mechanical properties of single walled carbon nanotubes using a spring based finite element approach, Computational Materials Science, 41(4), 561-569.

Han, T. W., He, P. F., Wang, J. A. and Wu, A. H. (2010). Molecular dynamics simulation of a single graphene sheet under tension, New Carbon Materials, 25(4), 261-266.

Hernández, E., Goze, C. and Rubio, A. (1998). Elastic Properties of $\mathrm{C}$ and $\mathrm{BxCyNz}$ composite nanotubes, Physical Review Letters, 80(20), 4502-4505.

Iijima, S. (1991). Helical microtubules of praphitic carbon, Nature, 354, 56-59.

Jiang, L., Cheung, R., Hedley, J., Hassan, M., Harris, A. J., Burdess, J. S., Mehregany, M. \& Zorman, C. A. (2006). Sic cantilever resonators with electrothermal actuation. Sensors and Actuators a-Physical, 128(2), 376-386.

Jin, Y. and Yuan, F. G. (2003). Simulation of elastic properties of single-walled carbon nanotubes, Composites Science and Technology, 63(11), 1507-1515.

Kalamkarov, A. L., Georgiades, A. V., Rokkam, S. K., Veedu, v. P. and Ghasemi-Nejhad, M. N. (2006). Analytical and numerical techniques to predict carbon nanotubes properties. International journal of solids and structures, 43, 6832-6854.

Kudin, K. N., Scuseria, G. E. and Yakobson, B. I. (2001). C2F, BN, and $C$ nanoshell elasticity from $a b$ initio computations, Physical Review $B$, 64(23).

Li, C. Y. and Chou, T. W. (2003a). A structural mechanics approach for the analysis of carbon nanotubes, International Journal of Solids and Structures, 40(10), 2487-2499.

Li, C. Y. and Chou, T. W. (2003b). Single-walled carbon nanotubes as ultrahigh frequency nanomechanical resonators, Physical Review B, 68(7). 
Li, L. and Chang, T. (2009). Explicit solution for $G$-band mode frequency of single-walled carbon nanotubes, Acta Mechanica Solida Sinica, 22(6).

Lourie, O. and Wagner, H. D. (1998). Evaluation of young's modulus of carbon nanotubes by microRaman spectrocopy, Journal of materials research, 13(9), 2418-2422.

Lu, J. P. (1997). Elastic Properties of carbon nanotubes and nanoropes, Physical Review Letters, 79(7), 1297-1300.

Maiti, A. (2008). Multiscale modeling with carbon nanotubes. Microelectronics Journal, 39 (2), 208-221.

Odegard, G. M., Gates, T. S., Nicholson, L. M. and Wise, K. E. (2002) Equivalent-continuum modeling of nano-structured materials, Composites Science and Technology, 62(14), 1869-1880.

Pantano, A., Parks, D. M. and Boyce, M. C. (2004) Mechanics of deformation of single- and multiwall carbon nanotubes, Journal of the Mechanics and Physics of Solids, 52(4), 789-821.

Sakhaee-Pour, A., Ahmadian, M. T. and Gerami, A. (2008) Development of an equation to predict radial modulus of elasticity for single-walled carbon nanotubes, Proceedings of the Institution of Mechanical Engineers Part C-Journal of Mechanical Engineering Science, 222(6), 11091115 .

Sears, A. and Batra, R. C. (2004) Macroscopic properties of carbon nanotubes from molecularmechanics simulations, Physical Review B, 69(235406).

Thostenson, E. T., Ren, Z. F. and Chou, T. W. (2001) Advances in the science and technology of carbon nanotubes and their composites: a review, Composites Science and Technology, 61(13), 1899-1912.

Tombler, T., Zhou, C., Alexseyev, L., Kong, J., Dai, H., Liu, L., Jayanthi, C. S., Tang, M. and Wu,
S.-Y. (2000) Reversible electromechanical characteristic of carbon nanotubes under localprobe manipulation, Nature, 405, 769 .

Tserpes, K. I. \& Papanikos, P. (2005) Finite element modeling of single-walled carbon nanotubes. Composites Part B-Engineering, 36, 468-477.

Tu, Z. C. and Ou-Yang, Z. (2002) Single-walled and multiwalled carbon nanotubes viewed as elastic tubes with the effective Young's moduli dependent on layer number, Physical Review B, $65(23)$.

Xin , Z., Jianjun, Z., Zhong-can and Ou-Yang (2000) Strain energy and Young's modulus of single-wall carbon nanotubes calculated from electronic energy-band theory, Physical Review B, 62(20), 13692-13696.

Yakobson, B. I., Brabec, C. J. and Bernholc, J. (1996) Nanomechanics of carbon tubes: Instabilities beyond Linear response, Physical Review Letters, 76(14), 2511-2514. 